\title{
Stochastic Stability for Time-Delay Markovian Jump Systems with Sector-Bounded Nonlinearities and More General Transition Probabilities
}

\author{
Dan Ye, ${ }^{1,2}$ Quan-Yong Fan, ${ }^{1}$ Xin-Gang Zhao, ${ }^{2}$ and Guang-Hong Yang $^{1}$ \\ ${ }^{1}$ College of Information Science and Engineering, Northeastern University, Shenyang, Liaoning 110189, China \\ ${ }^{2}$ State Key Laboratory of Robotics, Shenyang Institute of Automation, CAS, Shenyang, Liaoning 110016, China
}

Correspondence should be addressed to Dan Ye; yedan@ise.neu.edu.cn

Received 29 January 2013; Accepted 20 March 2013

Academic Editor: Xuejun Xie

Copyright (C) 2013 Dan Ye et al. This is an open access article distributed under the Creative Commons Attribution License, which permits unrestricted use, distribution, and reproduction in any medium, provided the original work is properly cited.

\begin{abstract}
This paper is concerned with delay-dependent stochastic stability for time-delay Markovian jump systems (MJSs) with sectorbounded nonlinearities and more general transition probabilities. Different from the previous results where the transition probability matrix is completely known, a more general transition probability matrix is considered which includes completely known elements, boundary known elements, and completely unknown ones. In order to get less conservative criterion, the state and transition probability information is used as much as possible to construct the Lyapunov-Krasovskii functional and deal with stability analysis. The delay-dependent sufficient conditions are derived in terms of linear matrix inequalities to guarantee the stability of systems. Finally, numerical examples are exploited to demonstrate the effectiveness of the proposed method.
\end{abstract}

\section{Introduction}

During the past decades, much attention has been devoted to the stochastic systems since stochastic modeling has an important science and engineering application $[1,2]$. As an important class of stochastic systems, MJSs have attracted a lot of interest, since they can be used to model many practical dynamical systems, such as power systems, manufacturing systems, and economic systems in which they may experience failure or repairs, abrupt environmental disturbances, and abrupt changes in the operating point of a nonlinear plant (see $[3,4])$. Theoretical results of this class of systems have been applied in many processes, such as target tracking, manufactory processes, solar thermal receivers, and faultdetection and fault-tolerant systems. Some examples can be found in [5-7].

For MJSs, the transition probabilities of the jumping process are important, but most of the previous issues on this kind of systems usually assumed that the elements of the transition probability matrix are completely known. Based on this condition, a lot of research results have been worked out in the literature, such as [8-13]. However, in many practical engineering applications, we cannot get all of the transition probabilities elements, and some elements might be time variable in some cases. Therefore, the study of Markov jump systems with partly known transition probabilities becomes necessary and some well-known results have been published, see [11-13]. In some cases, the exact value of some elements of the transition probability matrix can not be obtained, but their lower and upper bounds can be determined. The case has been addressed by [8], but its method cannot be used to deal with the case where there is no information available for transition probabilities. In [9], the more general partly known transition probabilities are investigated, which covers the cases that the transition probabilities are exactly known, unknown, and unknown but with known bounds. However, a conservative condition to relax inequality is used to deal with this kind of transition probabilities, and the time delay is not considered.

It is well known that time delay is very common in variously practical systems such as manufacturing systems, chemical processes, network control systems (NCSs), and telecommunication and economic systems. They are often the causes of oscillation, instability, and poor performance in 
many control systems. In the past years, time-delay system has been widely studied, and many analysis results have been reported (see $[14,15])$. However, many approaches for timedelay problems only employed partial information of the delay-related terms. The quadratic integral terms of the form $\int_{t-h(t)}^{t} f(\alpha) d(\alpha)$ have been the major choices to construct the Lyapunov-Krasovskii functional, ignoring the employment of the delay upper bound $\bar{h}$ (see [16]). Recently, Markovian systems with time delay have been widely studied (see [17-19]). But the time delays in $[17,19]$ are invariant, which is limited in the practical application. Although [18] considers the systems with time-varying delay, some useful terms are ignored to construct the Lyapunov-Krasovskii functional, which may lead to considerable conservativeness. Unfortunately, in the aforementioned papers about time delay, the elements of the considered transition probability matrix are completely known. To the best of the authors' knowledge, up to now, there are few papers concerning both time delay and more general transition probability to deal with stochastic stability for nonlinear Markov jump systems.

Motivated by the previous points, in this paper, we are concerned with the stochastic stability analysis for a class of nonlinear MJSs with time-varying delay and general transition probabilities. The considered nonlinearity is the socalled sector-bounded nonlinearity [20], which is quite more general than the usual Lipschitz conditions. By exploiting full information of the delay-related terms including $h(t)$ and $\bar{h}$ and inherent relationship between the elements in transition probabilities, less conservative stability criterions are obtained in the framework of LMIs. Numerical examples are given to demonstrate the superiorities and effectiveness of the proposed method.

Notations. The superscript $T$ stands for matrix transposition, and $R^{n}$ denotes the $n$ dimensional Euclidean space. $E[\cdot]$ stands for the $n$ mathematical expectation; $\mathrm{He}(X)$ represents the sum of $X$ and $X^{T}$. In addition, in symmetric block matrices or long matrix expressions, $*$ is used as an ellipsis for the terms that are introduced by symmetry. The notation $P>$ $0(\geq 0)$ means that $P$ is real symmetric positive (semipositive) definite. $\varepsilon[\cdot]$ stands for the expectation operator with respect to the given probability measure P. $L_{\mathscr{F}_{0}}^{2}\left([-\bar{h}, 0] ; \mathbb{R}^{n}\right)$ denotes the family of $\mathbb{R}^{n}$-valued stochastic process $x(t),-\bar{h} \leq t \leq 0$, such that $x(t)$ is $\mathscr{F}_{0}$-measurable and $\int_{-\bar{h}}^{0} E\left\{\|x(t)\|^{2}\right\} d t<\infty$. Matrices, if their dimensions are not explicitly stated, are assumed to be compatible for algebraic operations.

\section{Problem Statement}

Consider a class of stochastic nonlinear MJSs with sectorbounded nonlinearity and time-varying delay as follows:

$$
\begin{gathered}
\dot{x}(t)=A(r(t)) x(t)+A_{d}(r(t)) x(t-h(t)) \\
+f(t, x(t), r(t))+f_{d}(t, x(t-h(t)), r(t)), \\
x(t)=\psi(t), \quad-\bar{h} \leq t \leq 0,
\end{gathered}
$$

where $x(t) \in R^{n}$ is the state variable, and $A(r(t)) \in R^{n \times n}$ and $A_{d}(r(t)) \in R^{n \times n}$ are known matrix functions of the Markov jump process $r(t) . f(*)$ and $f_{d}(*)$ are the vectorvalued nonlinear functions. Also $h(t) \in[0, \bar{h}], \dot{h}(t) \leq \bar{\mu}$, and $\psi(t) \in L_{\mathscr{F}_{0}}^{2}\left([-\bar{h}, 0] ; \mathbb{R}^{n}\right) . r(t)$ is a time-homogeneous Markov process with right continuous trajectories and takes values on the finite set $\mathbb{L}=\{1,2, \ldots, \mathbb{N}\}$ with the following mode transition probabilities:

$$
\operatorname{Pr}\{r(t+d t)=j \mid r(t)=i\}= \begin{cases}\pi_{i j} d t+o(d t), & i \neq j \\ 1+\pi_{i i} d t+o(d t), & i=j\end{cases}
$$

where $d t>0$, where $\lim _{d t \rightarrow 0}(o(d t) / d t)=0 . \pi_{i j} \geq 0$, for $i \neq j$, is the transition rate from mode $i$ to mode $j$, and

$$
\sum_{j=1, i \neq j}^{\mathbb{N}} \pi_{i j}=-\pi_{i i}, \quad i=(1, \ldots, \mathbb{N}) .
$$

In this paper, more general transition probabilities of the jumping process in [9] are considered. Namely, some elements in matrix have been exactly known, some ones have been merely known with lower and upper bounds, and others may have no information to use. For instance, the transition probability matrix might be described by matrix

$$
\left[\begin{array}{ccccc}
\pi_{11} & ? & \pi_{13} & \cdots & \pi_{1 \mathbb{N}} \\
? & ? & \alpha & \cdots & \pi_{2 \mathbb{N}} \\
\vdots & \cdots & \cdots & \ddots & \vdots \\
\pi_{\mathbb{N} 1} & \beta & \cdots & \cdots & \pi_{\mathbb{N N}}
\end{array}\right]
$$

where "?" denotes the inaccessible elements, $\alpha$ and $\beta$ have known lower and upper bounds $(\underline{\alpha} \leq \alpha \leq \bar{\alpha}$ and $\underline{\beta} \leq \beta \leq \bar{\beta})$ and $\pi_{i j}$ is exactly known.

For notation clarity, for all $i, j \in \mathbb{L}$, we further rewrite $\mathbb{L}=\mathbb{L}_{k}^{i} \cup \mathbb{L}_{u k}^{i}$ with

$$
\begin{gathered}
\mathbb{L}_{k}^{i} \triangleq\left\{j: \underline{\pi}_{i j} \leq \pi_{i j} \leq \bar{\pi}_{i j}\right\}, \\
\mathbb{\mathbb { L }}_{u k}^{i} \triangleq\left\{j: j \notin \mathbb{\mathbb { L }}_{k}^{i}\right\}, \\
\mathscr{L}_{k}^{i} \triangleq\left\{m \mid m \in \mathbb{L}_{k}^{i}, m \neq i\right\}, \\
\mathscr{L}_{u k}^{i} \triangleq\left\{m \mid m \in \mathbb{\mathbb { L }}_{u k}^{i}, m \neq i\right\},
\end{gathered}
$$

where $\underline{\pi}_{i j}$ and $\bar{\pi}_{i j}$ are known lower and upper bounds of $\pi_{i j}$, respectively.

Remark 1. In order to get more general result, this paper considers that $\mathbb{L}_{k}^{i}$ includes two cases. One is that $\pi_{i j}$ is exactly known, and the other is that it is unknown but with upper and lower known bounds, which has been considered in [8]. If $\pi_{i j}$ is exactly known, it also can be seen as a rate with equal bounds.

The set $\mathbb{L}$ comprises the various operation modes of system (1), and for each possible value of $r(t)=i(i \in \mathbb{L})$, 
the matrices and the vector-valued nonlinear functions associated with the $i$ th mode will be denoted by

$$
\begin{gathered}
A_{i}=A(r(t)=i), \quad A_{d i}=A_{d}(r(t)=i), \\
f_{i}(t)=f(t, x(t), r(t)=i), \\
f_{d i}(t)=f_{d}(t, x(t-h(t)), r(t)=i) .
\end{gathered}
$$

In system (1), the vector-valued nonlinear functions $f(*)$ and $f_{d}(*)$ are assumed to satisfy the following sectorbounded conditions:

$$
\begin{aligned}
& {\left[f(x)-f(y)-M_{1}(x-y)\right]^{T}} \\
& \times\left[f(x)-f(y)-M_{2}(x-y)\right] \leq 0, \\
& {\left[f_{d}(x)-f_{d}(y)-N_{1}(x-y)\right]^{T}} \\
& \quad \times\left[f_{d}(x)-f_{d}(y)-N_{2}(x-y)\right] \leq 0,
\end{aligned}
$$

where for all $x, y \in R^{n}, M_{1}, M_{2}, N_{1}, N_{2} \in R_{n \times n}$ are known constant matrices. Without loss of generality, the following equations are always assumed:

$$
f(0)=0, \quad f_{d}(0)=0 .
$$

Remark 2. As in [20], the nonlinear functions $f(*), f_{d}(*)$ are said to belong to sectors. In other words, the nonlinearities are bounded by sectors. Further, both the filter design problems and control analysis have been investigated; see [21, 22]. The nonlinear descriptions in (7) are more general than the usual sigmoid functions and the recently commonly used Lipschitz conditions [21], and $M_{1}, M_{2}, N_{1}, N_{2}$ are lower and upper slope bounds, respectively.

Lemma 3. Assume that $f(*)$ is a vector-valued nonlinear function and $M_{1}, M_{2}$ are known constant matrices. If there exists

$$
\left[f(x)-M_{1} x\right]^{T}\left[f(x)-M_{2} x\right] \leq 0,
$$

it can be obtained that

$$
\left[\begin{array}{c}
x \\
f(x)
\end{array}\right]^{T}\left[\begin{array}{cc}
\bar{M}_{1} & \bar{M}_{2} \\
* & I
\end{array}\right]\left[\begin{array}{c}
x \\
f(x)
\end{array}\right] \leq 0,
$$

with $\bar{M}_{1}=\left(M_{1}^{T} M_{2}+M_{2}^{T} M_{1}\right) / 2, \bar{M}_{2}=-\left(M_{1}^{T}+M_{2}^{T}\right) / 2$.

Proof. Notice the fact that

$$
\begin{aligned}
& {\left[f(x)-M_{1} x\right]^{T}\left[f(x)-M_{2} x\right] \leq 0,} \\
& {\left[f(x)-M_{2} x\right]^{T}\left[f(x)-M_{1} x\right] \leq 0 ;}
\end{aligned}
$$

so,

$$
\begin{aligned}
0.5\left\{\left[f(x)-M_{1} x\right]^{T}\left[f(x)-M_{2} x\right]\right. \\
\left.\quad+\left[f(x)-M_{2} x\right]^{T}\left[f(x)-M_{1} x\right]\right\} \leq 0 .
\end{aligned}
$$

Then, (10) can be obtained. Thus, the proof is complete.
For the sake of simplicity, the solution $x\left(t, x_{0}, r_{0}\right)$ of system (1) with $r_{0} \in \mathbb{L}$ is denoted by $x(t)$. It is known that $\{x(t), r(t)\}$ is a Markov process with an initial state $\left(x_{0}, r_{0}\right)$, and its weak infinitesimal generator acting on function $V$ is defined as follows:

$$
\begin{gathered}
\xi V(x(t), r(t), t) \\
=\lim _{\Delta \rightarrow 0^{+}} \frac{1}{\Delta}[\varepsilon(V(x(t+\Delta), t+\Delta, r(t+\Delta)) \mid x(t), r(t)=i) \\
-V(x(t), r(t), t)] .
\end{gathered}
$$

Throughout this paper, the following definition is used.

Definition 4. System (1) is said to be stochastically stable if for finite $\psi(t) \in R^{n}$ defined on $[-\bar{h}, 0]$ and $r(0) \in \mathbb{L}$, the following is satisfied:

$$
\lim _{t \rightarrow \infty} \varepsilon\left\{\int_{0}^{t} x^{T}(s, \psi, r(0)) x(s, \psi, r(0)) d s\right\}<\infty
$$

where $x(s, \psi, r(0))$ denotes the solution to system at time $t$ under the initial conditions $\psi(t)$ and $r(0)$.

\section{Stochastic Stability Analysis}

In this section, new LMI-based sufficient conditions for system (1) will be presented based on a special LyapunovKrasovskii functional and Lemma 3.

For presentation convenience, it is necessary to define

$$
\begin{gathered}
\chi_{i}(t)=\left[x^{T}(t), x^{T}(t-h(t)), x^{T}(t-\bar{h}),\right. \\
\left.\dot{x}^{T}(t), f_{i}^{T}(t), f_{d i}^{T}(t)\right]^{T} \\
=e_{1} x(t)+e_{2} x(t-h(t))+e_{3} x(t-\bar{h}) \\
+e_{4} \dot{x}(t)+e_{5} f_{i}(t)+e_{6} f_{d i}(t),
\end{gathered}
$$

and the corresponding block entry matrices are $e_{j}, j \in$ $\{1, \ldots, 6\}$. In addition, there are some other definitions. Consider

$$
\begin{gathered}
\lambda_{k}^{i}=\sum_{m \in \mathscr{L}_{k}^{i}} \pi_{i m}, \quad \underline{\lambda}_{k}^{i}=\sum_{m \in \mathscr{L}_{k}^{i}} \underline{\pi}_{i m}, \quad \lambda_{u k}^{i}=\sum_{m \in \mathscr{L}_{u k}^{i}} \pi_{i m}, \\
\mathscr{P}_{k}^{i}=\sum_{m \in \mathscr{L}_{k}^{i}} \pi_{i j} P_{m}, \quad \overline{\mathscr{P}}_{k}^{i}=\sum_{m \in \mathscr{L}_{k}^{i}} \bar{\pi}_{i j} P_{m},
\end{gathered}
$$

Theorem 5. For given scalars $\bar{h}>0$ and $\bar{\mu}$, the delayed system (1) with $\dot{h}(t) \leq \bar{\mu}$ is stochastically stable if there exist scalars $\eta_{1 i}>0$ and $\eta_{2 i}>0$ and matrices $P_{i}>0, Q_{0 i}>0, Q_{1 i}>0$, 
$S_{0}>0, S_{1}>0, R>0, Y_{11}^{i}, Y_{12}^{i}, Y_{22}^{i}, Z_{11}^{i}, Z_{12}^{i}, Z_{22}^{i}, \Phi_{i}, H_{1 i}$, and $\mathrm{H}_{2 i}$ such that the following conditions hold:

$$
\begin{aligned}
H e & \left(\Phi_{i}\left[A_{i} e_{1}^{T}+A_{d i} e_{2}^{T}-e_{4}^{T}+e_{5}^{T}+e_{6}^{T}\right]\right. \\
& \left.+Z_{12}^{i}\left(e_{2}-e_{3}\right)^{T}+Y_{12}^{i}\left(e_{1}-e_{2}\right)^{T}\right) \\
+ & H e\left(e_{4} P_{i} e_{1}^{T}\right)-e_{3} Q_{0 i} e_{3}^{T}+\bar{h} e_{4}\left(S_{0}+S_{1}\right) e_{4}^{T} \\
- & (1-\bar{\mu}) e_{2} Q_{1 i} e_{2}^{T}+\bar{h} Y_{11}^{i}+e_{1}\left(\bar{h} R+Q_{0 i}+Q_{1 i}+\Psi_{i}\right) e_{1}^{T} \\
- & \eta_{1 i}\left(e_{1} \bar{M}_{1 i} e_{1}^{T}+e_{5} \bar{M}_{2 i}^{T} e_{1}^{T}+e_{1} \bar{M}_{2 i} e_{5}^{T}+e_{5} e_{5}^{T}\right) \\
- & \eta_{2 i}\left(e_{2} \bar{N}_{1 i} e_{2}^{T}+e_{6} \bar{N}_{2 i}^{T} e_{2}^{T}+e_{2} \bar{N}_{2 i} e_{6}^{T}+e_{6} e_{6}^{T}\right)<0,
\end{aligned}
$$

$$
\begin{aligned}
& \operatorname{He}\left(\Phi_{i}\left[A_{i} e_{1}^{T}+A_{d i} e_{2}^{T}-e_{4}^{T}+e_{5}^{T}+e_{6}^{T}\right]\right. \\
& \left.+Z_{12}^{i}\left(e_{2}-e_{3}\right)^{T}+Y_{12}^{i}\left(e_{1}-e_{2}\right)^{T}\right) \\
& +\mathrm{He}\left(e_{4} P_{i} e_{1}^{T}\right)-e_{3} Q_{0 i} e_{3}^{T}+\bar{h} e_{4} S_{0} e_{4}^{T}-(1-\bar{\mu}) e_{2} Q_{1 i} e_{2}^{T} \\
& +\bar{h} Z_{11}^{i}+e_{1}\left(\bar{h} R+Q_{0 i}+Q_{1 i}+\Psi_{i}\right) e_{1}^{T} \\
& -\eta_{1 i}\left(e_{1} \bar{M}_{1 i} e_{1}^{T}+e_{5} \bar{M}_{2 i}^{T} e_{1}^{T}+e_{1} \bar{M}_{2 i} e_{5}^{T}+e_{5} e_{5}^{T}\right) \\
& -\eta_{2 i}\left(e_{2} \bar{N}_{1 i} e_{2}^{T}+e_{6} \bar{N}_{2 i}^{T} e_{2}^{T}+e_{2} \bar{N}_{2 i} e_{6}^{T}+e_{6} e_{6}^{T}\right)<0, \\
& {\left[\begin{array}{cc}
Y_{11}^{i} & Y_{12}^{i} \\
* & Y_{22}^{i}
\end{array}\right] \geq 0, \quad\left[\begin{array}{cc}
Z_{11}^{i} & Z_{12}^{i} \\
* & Z_{22}^{i}
\end{array}\right] \geq 0,} \\
& S_{0} \geq Z_{22}^{i}, \quad S_{0}+\left(1-\bar{\mu} S_{1}\right) \geq Y_{22}^{i}, \\
& i \in \mathbb{L}_{k}^{i}\left\{\begin{array}{l}
\sum_{m \in \mathscr{L}_{k}^{i}} \bar{\pi}_{i m}\left(Q_{0 m}+Q_{1 m}\right)+\bar{\pi}_{i i}\left(Q_{0 i}+Q_{1 i}\right) \\
-\left(\underline{\lambda}_{k}^{i}+\underline{\pi}_{i i}\right)\left(Q_{0 j}+Q_{1 j}\right)-R<0, \quad j \in \mathbb{L}_{u k}^{i}, \\
\sum_{m \in \mathscr{L}_{k}^{i}} \bar{\pi}_{i m} Q_{0 m}+\bar{\pi}_{i i} Q_{0 i}-\left(\underline{\lambda}_{k}^{i}+\underline{\pi}_{i i}\right) Q_{0 j}-R<0, \\
\quad j \in \mathbb{L}_{u k}^{i},
\end{array}\right.
\end{aligned}
$$

$$
i \in \mathbb{L}_{u k}^{i}\left\{\begin{array}{l}
\sum_{m \in \mathbb{L}_{k}^{i}} \bar{\pi}_{i m}\left(Q_{0 m}+Q_{1 m}\right)-\sum_{m \in \mathbb{L}_{k}^{i}} \underline{\pi}_{i m} H_{2 i}-R<0, \\
\sum_{m \in \mathbb{\pi}_{k}^{i}}^{i} \bar{\pi}_{i m} Q_{0 m}-\sum_{m \in \mathbb{L}_{k}^{i}} \underline{\pi}_{i m} H_{2 i}-R<0, \\
Q_{0 j}+Q_{1 j}-H_{2 i} \leq 0, \quad j \in \mathbb{L}_{u k}^{i}, j \neq i, \\
Q_{0 j}-H_{2 i} \geq 0, \quad j \in \mathbb{L}_{u k}^{i}, j=i, \\
P_{j}-H_{1 i} \leq 0, \quad j \in \mathbb{L}_{u k}^{i}, j \neq i, \\
P_{j}-H_{1 i} \geq 0, \quad j \in \mathbb{L}_{u k}^{i}, j=i,
\end{array}\right.
$$

where

$$
\Psi_{i}= \begin{cases}\overline{\mathscr{P}}_{k}^{i}+\bar{\pi}_{i i} P_{i}-\left(\underline{\pi}_{i i}+\underline{\lambda}_{k}^{i}\right) P_{l}, & i \in \mathbb{L}_{k}^{i}, l \in \mathbb{L}_{u k}^{i}, \\ \overline{\mathscr{P}}_{k}^{i}-\sum_{m \in \mathbb{L}_{k}^{i}} \underline{\pi}_{i m} H_{1 i}, & i \in \mathbb{L}_{u k}^{i},\end{cases}
$$

$$
\begin{array}{ll}
\bar{M}_{1}=\frac{\left(M_{1}^{T} M_{2}+M_{2}^{T} M_{1}\right)}{2}, & \bar{M}_{2}=-\frac{\left(M_{1}^{T}+M_{2}^{T}\right)}{2}, \\
\bar{N}_{1}=\frac{\left(N_{1}^{T} N_{2}+N_{2}^{T} N_{1}\right)}{2}, & \bar{N}_{2}=-\frac{\left(N_{1}^{T}+N_{2}^{T}\right)}{2},
\end{array}
$$

Proof. First, in order to cast the model involved into the framework of the Markov processes, a new process is defined as $\left\{\left(x_{t}, r(t)\right), t \geq 0\right\}$ by

$$
x_{t}(s)=x(t+s), \quad-2 \bar{h} \leq s \leq 0 ;
$$

so, $\left\{\left(x_{t}, r(t)\right), t \geq \bar{h}\right\}$ is a Markov process with initial state $(\psi(\cdot), r(0))$.

Then, the stochastic Lyapunov-Krasovskii functional is chosen as follows:

$$
V\left(x_{t}, r(t), t\right)=\sum_{j=1}^{6} V_{j}\left(x_{t}, r(t), t\right)
$$

where

$$
\begin{aligned}
V_{1}\left(x_{t}, r(t), t\right) & =x^{T}(t) P(r(t)) x(t), \\
V_{2}\left(x_{t}, r(t), t\right) & =\int_{t-\bar{h}}^{t} x^{T}(\alpha) Q_{0}(r(t)) x(\alpha) d \alpha, \\
V_{3}\left(x_{t}, r(t), t\right) & =\int_{t-h(t)}^{t} x^{T}(\alpha) Q_{1}(r(t)) x(\alpha) d \alpha, \\
V_{4}\left(x_{t}, r(t), t\right) & =\int_{-\bar{h}}^{0} \int_{t+\beta}^{t} \dot{x}^{T}(\alpha) S_{0} \dot{x}(\alpha) d \alpha d \beta, \\
V_{5}\left(x_{t}, r(t), t\right) & =\int_{-h(t)}^{0} \int_{t+\beta}^{t} \dot{x}^{T}(\alpha) S_{1} \dot{x}(\alpha) d \alpha d \beta, \\
V_{6}\left(x_{t}, r(t), t\right) & =\int_{-\bar{h}}^{0} \int_{t+\beta}^{t} x^{T}(\alpha) R x(\alpha) d \alpha d \beta, \\
P(r(t))>0, \quad Q_{0}(r(t))>0, \quad Q_{1}(r(t))>0, & S_{0}>0, \quad S_{1}>0, \quad R>0 .
\end{aligned}
$$

The infinitesimal generator of the Markov process acting on $V(\cdot)$ and emanating from the point $r(t)=i(i \in \mathbb{L})$ is given as follows:

$$
\begin{aligned}
\xi V & \left(x_{t}, r(t), t\right) \\
= & 2 \chi_{i}^{T}(t) e_{4} P_{i} e_{1}^{T} \chi_{i}(t) \\
& +\chi_{i}^{T}(t)\left[e_{1} \sum_{j=1}^{\mathbb{N}} \pi_{i j} P_{j} e_{1}^{T}+e_{1} Q_{0 i} e_{1}^{T}-e_{3} Q_{0 i} e_{3}^{T}\right] \chi_{i}(t) \\
& +\int_{t-\bar{h}}^{t} x^{T}(\alpha) \sum_{j=1}^{\mathbb{N}} \pi_{i j} Q_{0 j} x(\alpha) d \alpha
\end{aligned}
$$




$$
\begin{aligned}
& +\chi_{i}^{T}(t)\left[e_{1} Q_{1 i} e_{1}^{T}-(1-\dot{h}(t)) e_{2} Q_{1 i} e_{2}^{T}\right] \chi_{i}(t) \\
& +\int_{t-h(t)}^{t} x^{T}(\alpha) \sum_{j=1}^{\mathbb{N}} \pi_{i j} Q_{1 j} x(\alpha) d \alpha \\
& +\bar{h} \chi_{i}^{T}(t) e_{4} S_{0} e_{4}^{T} \chi_{i}(t) \\
& -\int_{t-\bar{h}}^{t} \dot{x}^{T}(\alpha) S_{0} \dot{x}(\alpha) d \alpha+h(t) \chi_{i}^{T}(t) e_{4} S_{1} e_{4}^{T} \chi_{i}(t) \\
& -(1-\dot{h}(t)) \int_{t-h(t)}^{t} \dot{x}^{T}(\alpha) S_{1} \dot{x}(\alpha) d \alpha \\
& +\bar{h} \chi_{i}^{T}(t) e_{1} \operatorname{Re}_{1}^{T} \chi_{i}(t)-\int_{t-\bar{h}}^{t} x^{T}(\alpha) R x(\alpha) d \alpha .
\end{aligned}
$$

Then, choose

$$
\left[\begin{array}{cc}
Y_{11}^{i} & Y_{12}^{i} \\
* & Y_{22}^{i}
\end{array}\right] \geq 0, \quad\left[\begin{array}{cc}
Z_{11}^{i} & Z_{12}^{i} \\
* & Z_{22}^{i}
\end{array}\right] \geq 0
$$

it follows that

$$
\begin{aligned}
& \int_{t-h(t)}^{t}\left[\begin{array}{l}
\chi_{i}(t) \\
\dot{x}(\alpha)
\end{array}\right]^{T}\left[\begin{array}{cc}
Y_{11}^{i} & Y_{12}^{i} \\
* & Y_{22}^{i}
\end{array}\right]\left[\begin{array}{l}
\chi_{i}(t) \\
\dot{x}(\alpha)
\end{array}\right] d \alpha \\
& =\chi_{i}^{T}(t)\left[H e\left(Y_{12}^{i}\left(e_{1}-e_{2}\right)^{T}\right)+h(t) Y_{11}^{i}\right] \chi_{i}(t) \\
& +\int_{t-h(t)}^{t} \dot{x}^{T}(\alpha) Y_{22} \dot{x}(\alpha) d \alpha \geq 0, \\
& \int_{t-\bar{h}}^{t-h(t)}\left[\begin{array}{l}
\chi_{i}(t) \\
\dot{x}(\alpha)
\end{array}\right]^{T}\left[\begin{array}{cc}
Z_{11}^{i} & Z_{12}^{i} \\
* & Z_{22}^{i}
\end{array}\right]\left[\begin{array}{l}
\chi_{i}(t) \\
\dot{x}(\alpha)
\end{array}\right] d \alpha \\
& =\chi_{i}^{T}(t)\left[H e\left(Z_{12}^{i}\left(e_{2}-e_{3}\right)^{T}\right)+(\bar{h}-h(t)) Z_{11}^{i}\right] \chi_{i}(t) \\
& +\int_{t-\bar{h}}^{t-h(t)} \dot{x}^{T}(\alpha) Z_{22} \dot{x}(\alpha) d \alpha \geq 0 .
\end{aligned}
$$

And the constraints of the model dynamics can be written as $\chi_{i}^{T}(t) \Phi_{i}\left[A_{i} e_{1}^{T}+A_{d i} e_{2}^{T}-e_{4}^{T}+e_{5}^{T}+e_{6}^{T}\right] \chi_{i}(t) \equiv 0$ by introducing free variables $\Phi_{i}$. In addition, according to Lemma 3 and choosing scalar $\eta_{1 i}>0, \eta_{2 i}>0$, the following inequalities hold:

$-\eta_{1 i} \chi_{i}^{T}(t)\left(e_{1} \bar{M}_{1 i} e_{1}^{T}+e_{5} \bar{M}_{2 i}^{T} e_{1}^{T}+e_{1} \bar{M}_{2 i} e_{5}^{T}+e_{5} e_{5}^{T}\right) \chi_{i}(t) \geq 0$,

$-\eta_{2 i} \chi_{i}^{T}(t)\left(e_{2} \bar{N}_{1 i} e_{2}^{T}+e_{6} \bar{N}_{2 i}^{T} e_{2}^{T}+e_{2} \bar{N}_{2 i} e_{6}^{T}+e_{6} e_{6}^{T}\right) \chi_{i}(t) \geq 0$.

So, $\xi V(x(t), r(t), t)$ can be upper bounded as

$$
\begin{aligned}
& \xi V\left(x_{t}, r(t), t\right) \\
& \quad \leq \chi_{i}^{T}(t)\left\{\Omega_{1}(h(t))+e_{1} \sum_{j=1}^{\mathbb{N}} \pi_{i j} P_{j} e_{1}^{T}\right\} \chi_{i}(t)+\Omega_{2}+\Omega_{3},
\end{aligned}
$$

where

$$
\begin{aligned}
& \Omega_{1}(h(t))=h(t)\left(Y_{11}^{i}+e_{4} S_{1} e_{4}^{T}\right)+(\bar{h}-h(t)) Z_{11}^{i} \\
& +H e\left[\Phi_{i}\left(A_{i} e_{1}^{T}+A_{d i} e_{2}^{T}-e_{4}^{T}+e_{5}^{T}+e_{6}^{T}\right)\right. \\
& \left.+e_{4} P_{i} e_{1}^{T}+Y_{12}^{i}\left(e_{1}-e_{2}\right)^{T}+Z_{12}^{i}\left(e_{2}-e_{3}\right)^{T}\right] \\
& +\bar{h} e_{4} S_{0} e_{4}^{T}-e_{3} Q_{0 i} e_{3}^{T}-(1-\bar{\mu}) e_{2} Q_{1 i} e_{2}^{T} \\
& +e_{1}\left(Q_{0 i}+Q_{1 i}+\bar{h} R\right) e_{1}^{T} \\
& -\eta_{1 i}\left(e_{1} \bar{M}_{1 i} e_{1}^{T}+e_{5} \bar{M}_{2 i}^{T} e_{1}^{T}+e_{1} \bar{M}_{2 i} e_{5}^{T}+e_{5} e_{5}^{T}\right) \\
& -\eta_{2 i}\left(e_{2} \bar{N}_{1 i} e_{2}^{T}+e_{6} \bar{N}_{2 i}^{T} e_{2}^{T}+e_{2} \bar{N}_{2 i} e_{6}^{T}+e_{6} e_{6}^{T}\right), \\
& \Omega_{2}=-\int_{t-\bar{h}}^{t-h(t)} \dot{x}^{T}(\alpha)\left(S_{0}-Z_{22}^{i}\right) \dot{x}(\alpha) d \alpha \\
& -\int_{t-h(t)}^{t} \dot{x}^{T}(\alpha)\left(S_{0}-Y_{22}^{i}+(1-\bar{\mu}) S_{1}\right) \dot{x}(\alpha) d \alpha, \\
& \Omega_{3}=\int_{t-h(t)}^{t} x^{T}(\alpha)\left[\sum_{j=1}^{\mathbb{N}} \pi_{i j}\left(Q_{0 j}+Q_{1 j}\right)-R\right] x(\alpha) d \alpha \\
& +\int_{t-\bar{h}}^{t-h(t)} x^{T}(\alpha)\left[\sum_{j=1}^{\mathbb{N}} \pi_{i j} Q_{0 j}-R\right] x(\alpha) d \alpha .
\end{aligned}
$$

Since $\Omega_{1}(h(t))$ is a convex combination of the matrices $Y_{11}^{i}+e_{4} S_{1} e_{4}^{T}$ and $Z_{11}^{i}$ on $h(t)$, it can be handled nonconservatively by two corresponding boundary LMIs: one for $h(t)=\bar{h}$ and the other for $h(t)=0$.

So, $\Omega_{1}(h(t))<0$ is equivalent to

$$
\begin{aligned}
& \Omega_{1}(0)<0, \\
& \Omega_{1}(\bar{h})<0 .
\end{aligned}
$$

Then, the proof is separated into two cases, $i \in \mathbb{L}_{k}^{i}$ and $i \in \mathbb{L}_{u k}^{i}$.

Case $1\left(i \in \mathbb{L}_{k}^{i}\right)$. In this case, $\pi_{i i}$ is bounded or exactly known. Based on $\sum_{j=1}^{\mathbb{N}} \pi_{i j}=\lambda_{k}^{i}+\pi_{i i}+\lambda_{u k}^{i}=0$ and $-\sum_{l \in \mathscr{L}_{u k}^{i}} \pi_{i l} /\left(\pi_{i i}+\right.$ $\left.\lambda_{k}^{i}\right)=1$, it is easy to obtain that

$$
\begin{aligned}
& \Omega_{1}(h(t))+e_{1} \sum_{j=1}^{\mathbb{N}} \pi_{i j} P_{j} e_{1}^{T} \\
& =\Omega_{1}(h(t))+e_{1}\left(\sum_{j \in \mathscr{L}_{k}^{i}} \pi_{i j} P_{j}+\pi_{i i} P_{i}+\sum_{l \in \mathscr{L}_{u k}^{i}} \pi_{i l} P_{l}\right) e_{1}^{T}
\end{aligned}
$$




$$
\begin{aligned}
=-\frac{\sum_{l \in \mathscr{L}_{u k}^{i}} \pi_{i l}}{\pi_{i i}+\lambda_{k}^{i}}\left\{\Omega_{1}(h(t))\right. \\
\left.+e_{1}\left[\sum_{j \in \mathscr{L}_{k}^{i}} \pi_{i j} P_{j}+\pi_{i i} P_{i}-\left(\pi_{i i}+\lambda_{k}^{i}\right) P_{l}\right] e_{1}^{T}\right\} .
\end{aligned}
$$

Considering the fact that $0 \leq-\pi_{i l} /\left(\pi_{i i}+\lambda_{k}^{i}\right) \leq 1$, it is easy to obtain that $\Omega_{1}(h(t))+e_{1}\left[\sum_{j \in \mathscr{L}_{k}^{i}} \pi_{i j} P_{j}+\pi_{i i} P_{i}-\right.$ $\left.\left(\pi_{i i}+\lambda_{k}^{i}\right) P_{l}\right] e_{1}^{T}<0, l \in \mathbb{L}_{u k}^{i}$ can guarantee $\Omega_{1}(h(t))+$ $e_{1} \sum_{j=1}^{\mathbb{N}} \pi_{i j} P_{j} e_{1}^{T}<0$.

Since $\underline{\pi}_{i j} \leq \pi_{i j} \leq \bar{\pi}_{i j}$, the following inequality can be obtained:

$$
\begin{gathered}
\Omega_{1}(h(t))+e_{1}\left[\sum_{j \in \mathscr{L}_{k}^{i}} \pi_{i j} P_{j}+\pi_{i i} P_{i}-\left(\pi_{i i}+\lambda_{k}^{i}\right) P_{l}\right] e_{1}^{T} \\
\leq \Omega_{1}(h(t))+e_{1}\left[\overline{\mathscr{P}}_{k}^{i}+\bar{\pi}_{i i} P_{i}-\left(\underline{\pi}_{i i}+\underline{\lambda}_{k}^{i}\right) P_{l}\right] e_{1}^{T} .
\end{gathered}
$$

So, if (17) and (18) hold, $\Omega_{1}(h(t))+e_{1} \sum_{j=1}^{\mathbb{N}} \pi_{i j} P_{j} e_{1}^{T}<0$ can be obtained.

Similarly, $(21) \Rightarrow \Omega_{3}<0$.

And $(20) \Rightarrow \Omega_{2}<0$.

Therefore, when (17)-(21) are satisfied, $\xi V\left(x_{t}, r(t), t\right)<0$.

Case $2\left(i \in \mathbb{L}_{u k}^{i}\right)$. Because $\sum_{j=1}^{\mathbb{N}} \pi_{i j}=0$, there exist

$$
\begin{gathered}
-x^{T}(t) \sum_{j=1}^{\mathbb{N}} \pi_{i j} H_{1 i} x(t)=0 \\
-\int_{t-h}^{t} x^{T}(\alpha) \sum_{j=1}^{\mathbb{N}} \pi_{i j} H_{2 i} x(\alpha) d \alpha=0 .
\end{gathered}
$$

Adding the left sides of (36) into (31), the inequality can be written as

$$
\xi V\left(x_{t}, r(t), t\right) \leq \chi^{T}(t) \Gamma_{1}(h(t)) \chi(t)+\Gamma_{2}+\Gamma_{3},
$$

where

$$
\begin{aligned}
\Gamma_{1}(h(t))= & h(t)\left(Y_{11}^{i}+e_{4} S_{1} e_{4}^{T}\right)+(\bar{h}-h(t)) Z_{11}^{i} \\
& +H e\left[\Phi_{i}\left(A_{i} e_{1}^{T}+A_{d i} e_{2}^{T}-e_{4}^{T}+e_{5}^{T}+e_{6}^{T}\right)\right. \\
& \left.\quad+e_{4} P_{i} e_{1}^{T}+Y_{12}^{i}\left(e_{1}-e_{2}\right)^{T}+Z_{12}^{i}\left(e_{2}-e_{3}\right)^{T}\right] \\
& +\bar{h} e_{4} S_{0} e_{4}^{T}-e_{3} Q_{0 i} e_{3}^{T}-(1-\bar{\mu}) e_{2} Q_{1 i} e_{2}^{T} \\
& +e_{1}\left[Q_{0 i}+Q_{1 i}+\bar{h} R+\sum_{j \in \mathbb{L}_{k}^{i}} \pi_{i j}\left(P_{j}-H_{1 i}\right)\right] e_{1}^{T} \\
& +e_{1} \sum_{j \in \mathbb{L}_{u k}^{i}} \pi_{i j}\left(P_{j}-H_{1 i}\right) e_{1}^{T} \\
& -\eta_{1 i}\left(e_{1} \bar{M}_{1 i} e_{1}^{T}+e_{5} \bar{M}_{2 i}^{T} e_{1}^{T}+e_{1} \bar{M}_{2 i} e_{5}^{T}+e_{5} e_{5}^{T}\right) \\
& -\eta_{2 i}\left(e_{2} \bar{N}_{1 i} e_{2}^{T}+e_{6} \bar{N}_{2 i}^{T} e_{2}^{T}+e_{2} \bar{N}_{2 i} e_{6}^{T}+e_{6} e_{6}^{T}\right),
\end{aligned}
$$

$$
\begin{gathered}
\Gamma_{2}=\Omega_{2}, \\
\Gamma_{3}=\int_{t-h(t)}^{t} x^{T}(\alpha)\left[\sum_{j \in \mathbb{L}_{k}^{i}} \pi_{i j}\left(Q_{0 j}+Q_{1 j}-H_{2 i}\right)-R\right. \\
\left.+\sum_{j \in \mathbb{L}_{u k}^{i}} \pi_{i j}\left(Q_{0 j}+Q_{1 j}-H_{2 i}\right)\right] x(\alpha) d \alpha \\
+\int_{t-\bar{h}}^{t-h(t)} x^{T}(\alpha)\left[\sum_{j \in \mathbb{L}_{k}^{i}}^{\mathbb{N}} \pi_{i j}\left(Q_{0 j}-H_{2 i}\right)-R\right. \\
\left.+\sum_{j \in \mathbb{L}_{u k}^{i}}^{\mathbb{N}} \pi_{i j}\left(Q_{0 j}-H_{2 i}\right)\right] x(\alpha) d \alpha .
\end{gathered}
$$

So, $\Gamma_{1}(h(t))$ is also a convex combination of the matrices $Y_{11}^{i}+e_{4} S_{1} e_{4}^{T}$ and $Z_{11}^{i}$ on $h(t)$.

It is known that $\pi_{i i}<0$. At the same time, the boundary information $\underline{\pi}_{i j} \leq \pi_{i j} \leq \bar{\pi}_{i j}$ of the transition probabilities need to be used. It is clear that if (17)-(20) and (22) hold, $\xi V\left(x_{t}, r(t), t\right)<0$ can be achieved.

Taking into account (17)-(22), there exists a scalar $c>0$ for each $i \in \mathbb{L}$ such that

$$
\xi V(x(t), r(t), t) \leq-c\|x(t)\|^{2}
$$

which implies that system (1) is stochastically stable.

Therefore, the proof is completed.

If $f(t, x(t), r(t))=\Delta A(t, r(t)) x(t), f_{d}(t, x(t-h(t)), r(t))=$ $\Delta A_{d}(t, r(t)) x(t-h(t))$, system (1) becomes

$$
\begin{gathered}
\dot{x}(t)=(A(r(t))+\Delta A(t, r(t))) x(t) \\
+\left(A_{d}(r(t))+\Delta A_{d}(t, r(t))\right) x(t-h(t)), \\
x(t)=\psi(t), \quad-\bar{h} \leq t \leq 0,
\end{gathered}
$$

where $\Delta A(t, r(t)), \Delta A_{d}(t, r(t))$ are unknown time-varying matrices with appropriate dimensions and are assumed to be of the following form:

$$
\begin{aligned}
& {\left[\Delta A(t, r(t)) \quad \Delta A_{d}(t, r(t))\right]} \\
& =D(r(t)) E(t, r(t))\left[\begin{array}{lll}
F(r(t)) & F_{d}(r(t))
\end{array}\right],
\end{aligned}
$$

where $D(r(t)), F(r(t)), F_{d}(r(t))$ are known real constant matrices for all $r(t) \in \mathbb{L}$, and $E(t, r(t))$, for all $r(t) \in \mathbb{L}$, are the uncertain time-varying matrices satisfying

$$
E(t, r(t))^{T} E(t, r(t)) \leq I, \quad \forall r(t) \in \mathbb{L}
$$

It can be easily seen that system (40) is just the MJSs with norm bounded uncertainty (41). 
Choosing $p(t, r(t))=E(t, r(t)) q(t, r(t))$ and $q(t, r(t))=$ $F(r(t)) x(t)+F_{d}(r(t)) x(t-h(t))$, system (40) is equivalent to the following system:

$$
\begin{gathered}
\dot{x}(t)=A(r(t)) x(t)+A_{d}(r(t)) x(t-h(t)) \\
+D(r(t)) p(t, r(t)), \\
x(t)=\psi(t), \quad-\bar{h} \leq t \leq 0 .
\end{gathered}
$$

For each possible value of $r(t)=i(i \in \mathbb{L}), p(t, r(t))$, $D(r(t)), F(r(t))$, and $F_{d}(r(t))$ can be denoted as $p_{i}(t), D_{i}, F_{i}$, and $F_{d i}$. Then, it is necessary to choose $\chi_{i}(t)=\left[x^{T}(t), x^{T}(t-\right.$ $\left.h(t)), x^{T}(t-\bar{h}), \dot{x}^{T}(t), p_{i}^{T}(t)\right]^{T}$ and the corresponding block entry matrices as $e_{j}, j \in\{1, \ldots, 5\}$.

Then, the following corollary is obtained.

Corollary 6. If there exist matrices $P_{i}>0, Q_{0 i}>0, Q_{1 i}>0$, $S_{0}>0, S_{1}>0, R>0, Y_{11}^{i}, Y_{12}^{i}, Y_{22}^{i}, Z_{11}^{i}, Z_{12}^{i}, Z_{22}^{i}, \Phi_{i}, H_{1 i}$, and $\mathrm{H}_{2 i}$ such that (19)-(22) and the following LMIs are feasible for $i=1,2 \ldots, \mathbb{N}$ :

$$
\begin{aligned}
H e & \left(\Phi_{i}\left[A_{i} e_{1}^{T}+A_{d i} e_{2}^{T}-e_{4}^{T}+D_{i} e_{5}^{T}\right]\right. \\
& \left.+Z_{12}\left(e_{2}-e_{3}\right)^{T}+Y_{12}\left(e_{1}-e_{2}\right)^{T}\right)+H e\left(e_{4} P_{i} e_{1}^{T}\right) \\
- & e_{3} Q_{0 i} e_{3}^{T}+\bar{h} e_{4}\left(S_{0}+S_{1}\right) e_{4}^{T}-(1-\bar{\mu}) e_{2} Q_{1 i} e_{2}^{T} \\
+ & \bar{h} Y_{11}+e_{1}\left(\bar{h} R+Q_{0 i}+Q_{1 i}+\Psi_{i}\right) e_{1}^{T} \\
+ & \left(e_{1} F_{i}^{T}+e_{2} F_{d i}^{T}\right)\left(F_{i} e_{1}^{T}+F_{d i} e_{2}^{T}\right)-e_{5} e_{5}^{T}<0, \\
H e & \left(\Phi_{i}\left[A_{i} e_{1}^{T}+A_{d i} e_{2}^{T}-e_{4}^{T}+D_{i} e_{5}^{T}\right]\right. \\
& \left.+Z_{12}\left(e_{2}-e_{3}\right)^{T}+Y_{12}\left(e_{1}-e_{2}\right)^{T}\right) \\
+ & H e\left(e_{4} P_{i} e_{1}^{T}\right)-e_{3} Q_{0 i} e_{3}^{T}+\bar{h} e_{4} S_{0} e_{4}^{T}-(1-\bar{\mu}) e_{2} Q_{1 i} e_{2}^{T} \\
+ & \bar{h} Z_{11}+e_{1}\left(\bar{h} R+Q_{0 i}+Q_{1 i}+\Psi_{i}\right) e_{1}^{T} \\
+ & \left(e_{1} F_{i}^{T}+e_{2} F_{d i}^{T}\right)\left(F_{i} e_{1}^{T}+F_{d i} e_{2}^{T}\right)-e_{5} e_{5}^{T}<0,
\end{aligned}
$$

then the delayed uncertain system (40) with partly known transition probabilities is stochastically stable.

Proof. Here, the constraints of the model dynamics can be written as $\chi_{i}^{T}(t) \Phi_{i}\left[A_{i} e_{1}^{T}+A_{d i} e_{2}^{T}-e_{4}^{T}+D_{i} e_{5}^{T}\right] \chi_{i}(t) \equiv 0$. And the additional uncertainty constraint $(42)$ is given as follows:

$$
\begin{aligned}
0 & \leq q^{T}(t) q(t)-p^{T}(t) p(t) \\
& =\chi_{i}^{T}(t)\left\{\left(e_{1} F_{i}^{T}+e_{2} F_{d i}^{T}\right)\left(F_{i} e_{1}^{T}+F_{d i} e_{2}^{T}\right)-e_{5} e_{5}^{T}\right\} \chi_{i}(t) .
\end{aligned}
$$

Choose the Lyapunov functional as (25); so,

$$
\begin{aligned}
\xi V(x(t), r(t), t) \leq & \xi V(x(t), r(t), t) \\
& +q^{T}(t) q(t)-p^{T}(t) p(t) .
\end{aligned}
$$

TABLE 1: Comparison of maximum allowed $\bar{h}$.

\begin{tabular}{lllc}
\hline$\pi_{11}$ & -0.1 & -0.5 & -1 \\
\hline $\bar{h}$ by [16] & 0.4261 & 0.4206 & 0.4161 \\
$\bar{h}$ by [18] & 0.5050 & 0.4887 & 0.4855 \\
$\bar{h}$ by Theorem 5 & 0.5575 & 0.5499 & 0.5355 \\
\hline
\end{tabular}

TABLE 2: Maximum allowed $\bar{h}$ by Corollary 6.

\begin{tabular}{lccc}
\hline $\bar{\mu}$ & 0 & 0.2 & 0.6 \\
\hline $\bar{h}$ by Corollary 6 & 1.1739 & 1.0161 & 0.8618 \\
\hline
\end{tabular}

TABLE 3: Maximum allowed $\bar{h}$ by Theorem 5 .

\begin{tabular}{lccc}
\hline $\bar{\mu}$ & 0 & 0.2 & 0.6 \\
\hline $\bar{h}$ by Theorem 5 & 1.2999 & 1.0500 & 0.9236
\end{tabular}

Use the same method as the proof of Theorem 5 to make $\xi V(x(t), r(t), t)+q^{T}(t) q(t)-p^{T}(t) p(t)<0$, and then Corollary 6 can be obtained.

\section{Numerical Examples}

In this section, numerical examples are given to demonstrate the benefits and effectiveness of the proposed methods.

First, Example 1 in [16] is given to show the superiority of Theorem 5 for MJS (1) with no nonlinear terms and completely known transition probability matrix.

Example 7. Consider MJS (1) with $f(t, x(t), r(t))=0$ and $f_{d}(t, x(t-h(t)), r(t))=0$. The two modes are given as

$$
\begin{array}{ll}
A_{1}=\left[\begin{array}{ll}
-3.4888 & 0.8057 \\
-0.6451 & -3.2684
\end{array}\right], & A_{2}=\left[\begin{array}{cc}
-2.4898 & 0.2895 \\
1.3396 & -0.0211
\end{array}\right], \\
A_{d 1}=\left[\begin{array}{ll}
-0.8620 & -1.2919 \\
-0.6841 & -2.0729
\end{array}\right], & A_{d 2}=\left[\begin{array}{cc}
-2.8306 & 0.4978 \\
-0.8436 & -1.0115
\end{array}\right] .
\end{array}
$$

Supposing that $\mu=0.8$ and $\pi_{22}=-0.8$, compare the stochastic stability result in Theorem 5 with the stability theorem in $[16,18]$. For given $\pi_{11}$, the maximum $\bar{h}$, which satisfies the LMIs in (17)-(22), can be calculated.

Table 1 presents the comparison results, which shows that the stochastic stability result in Theorem 5 is less conservative than those in $[16,18]$ when no nonlinear terms exist and transition probabilities are completely known.

Next numerical example is for uncertain MJS (43) with more general transition probability. 
Example 8. Consider the following MJS (43) with four modes, whose system matrices are

$$
\begin{gathered}
A_{1}=\left[\begin{array}{cc}
-2.2460 & -1.4410 \\
-1.5937 & -2.9289
\end{array}\right], \quad A_{2}=\left[\begin{array}{cc}
-1.8999 & 0.8156 \\
-0.6900 & -0.7881
\end{array}\right], \\
A_{3}=\left[\begin{array}{cc}
-0.7523 & 0.7500 \\
1.5630 & -2.3540
\end{array}\right], \quad A_{4}=\left[\begin{array}{cc}
-1.7840 & 0.3640 \\
1.3670 & -1.5640
\end{array}\right], \\
A_{d 1}=\left[\begin{array}{cc}
-0.5 & -0.5 \\
0.05 & 0.01
\end{array}\right], \quad A_{d 2}=\left[\begin{array}{cc}
0.01 & 0 \\
0.05 & -0.01
\end{array}\right], \\
A_{d 3}=\left[\begin{array}{cc}
-0.01 & -0.02 \\
0.05 & 0.01
\end{array}\right], \quad A_{d 4}=\left[\begin{array}{cc}
0.03 & 0.01 \\
-0.2 & -0.1
\end{array}\right], \\
F_{1}=F_{2}=F_{3}=F_{4}=\left[\begin{array}{ll}
1 & 0
\end{array}\right], \\
F_{d 1}=F_{d 2}=F_{d 3}=F_{d 4}=\left[\begin{array}{ll}
-5 & 0.5
\end{array}\right], \\
D_{1}=D_{2}=D_{3}=D_{4}=\left[\begin{array}{l}
0.1 \\
0.1
\end{array}\right] .
\end{gathered}
$$

The more general transition probability matrix is chosen as follows, which includes completely known elements, boundary known elements, and completely unknown elements:

$$
\left[\begin{array}{cccc}
-1.3 & 0.2 & ? & ? \\
? & ? & 0.3 & 0.3 \\
0.6 & ? & -1.5 & ? \\
? & ? & ? & \alpha
\end{array}\right]
$$

Choose $-1.5 \leq \alpha \leq-0.9$. According to Corollary 6, Table 2 can be obtained for different values of $\bar{\mu}$. Also, the uncertain MJS (43) is stochastically stable. Moreover, as the upper bound of $\dot{h}(t)$, that is, $\bar{\mu}$, increases, the maximum allowed upper bound of $h(t)$ may be reduced.

Furthermore, a nonlinear MJS with sector-bounded nonlinearity is given.

Example 9. Consider MJS (1) with four models, whose $A_{i}, A_{d i}$ and the transition probability matrix are the same matrices as the ones of Example 8. The nonlinear functions are given as follows:

$$
\begin{aligned}
f_{1}(t) & =\left[\begin{array}{c}
0.1 x_{1}(t) \cos ^{2}\left(x_{1}(t)\right)-0.05\left(x_{1}(t)-x_{2}(t)\right) \\
-0.05 x_{1}(t)-0.1 x_{2}(t)
\end{array}\right], f_{2}(t) \\
& =2 f_{1}(t), f_{3}(t) \\
& =\left[\begin{array}{c}
0.15 x_{2}(t) \cos ^{2}\left(x_{1}(t)+x_{2}(t)\right)+0.1 x_{1}(t)+0.25 x_{2}(t) \\
0.15 x_{1}(t)+0.05 x_{2}(t)
\end{array}\right], f_{4}(t) \\
& =2 f_{3}(t), f_{d 1}(t) \\
& =f_{d 2}(t)=f_{d 3}(t)=f_{d 4}(t) \\
& =\left[\begin{array}{c}
0.12 x_{1}(t-h(t)) \sin ^{2}\left(x_{2}(t-h(t))\right)+0.04 x_{1}(t-h(t))+0.08 x_{2}(t-h(t)) \\
0.04 x_{1}(t-h(t))+0.04 x_{2}(t-h(t))
\end{array}\right] .
\end{aligned}
$$

According to (7), the corresponding matrices, which describe the sector bound, can be chosen as

$$
\begin{gathered}
M_{11}=\left[\begin{array}{ll}
0.05 & 0.05 \\
0.05 & 0.05
\end{array}\right], \quad M_{12}=2 M_{11}, \\
M_{13}=\left[\begin{array}{cc}
0.1 & 0.1 \\
0.1 & -0.05
\end{array}\right], \quad M_{14}=2 M_{13}, \\
M_{21}=\left[\begin{array}{cc}
-0.05 & 0.05 \\
-0.15 & -0.25
\end{array}\right], \quad M_{22}=2 M_{21}, \\
M_{23}=\left[\begin{array}{ll}
0.1 & 0.25 \\
0.2 & 0.15
\end{array}\right], \quad M_{24}=2 M_{23}, \\
N_{11}=N_{12}=N_{13}=N_{14}=\left[\begin{array}{cc}
0.04 & 0.08 \\
-0.04 & 0.12
\end{array}\right], \\
N_{21}=N_{22}=N_{23}=N_{24}=\left[\begin{array}{cc}
0.16 & 0.08 \\
0.12 & -0.04
\end{array}\right] .
\end{gathered}
$$

Choose $-1.5 \leq \alpha \leq-1$. Using Matlab LMI toolbox and Theorem 5, the maximum allowed upper bound of $h(t)$ can be obtained for different values of $\bar{\mu}$.

From Table 3, it can be seen that the stochastic stability can be guaranteed for nonlinear MJS (1) when more general transition probabilities (4) are considered.

\section{Conclusion}

This paper has presented a new method for solving the stochastic stability analysis problem for MJS with timevarying delay and sector-bounded nonlinearity. A general transition probability matrix is adopted, which includes completely known elements, boundary known elements, and completely unknown elements. Based on a new modedependent stochastic Lyapunov-Krasovskii functional and some new techniques to deal with transition probabilities, sufficient conditions with less conservativeness are derived in terms of LMIs to obtain stochastic stability of systems. 
Numerical examples have also demonstrated the effectiveness of the results.

\section{Acknowledgments}

This work is supported by the National Natural Science Foundation of China (nos. 61273155, 61273355, 61273356, 61035005), New Century Excellent Talents in University (no. NCET-11-0083), Foundation for the Author of National Excellent Doctoral Dissertation of China (no. 201157), the Foundation of State Key Laboratory of Robotics (no. 2012001).

\section{References}

[1] W. Zhang and L. Xie, "Interval stability and stabilization of linear stochastic systems," IEEE Transactions on Automatic Control, vol. 54, no. 4, pp. 810-815, 2009.

[2] X. J. Xie and L. Liu, "A homogeneous domination approach to state feedback of stochastic high-Order nonlinear systems with time-varying delay," IEEE Transactions on Automatic Control, vol. 58, pp. 494-495, 2013.

[3] E. F. Costa and J. B. R. do Val, "On the observability and detectability of continuous-time Markov jump linear systems," SIAM Journal on Control and Optimization, vol. 41, no. 4, pp. 1295-1314, 2002.

[4] W. Li, H. Su, D. Wei, and K. Wang, "Global stability of coupled nonlinear systems with Markovian switching," Communications in Nonlinear Science and Numerical Simulation, vol. 17, no. 6, pp. 2609-2616, 2012.

[5] Y. Long and G. H. Yang, "Fault detection for a class of nonhomogeneous Markov jump systems based on delta operator approach," Journal of Systems and Control Engineering, 2012.

[6] M. Mariton, Jump Linear Systems in Automatic Control, Marcel Dekker, New York, NY, USA, 1990.

[7] B. C. Willsky and A. S. Levy, "Stochastic stability research for complex power systems," Tech. Rep., Massachesetts Institute of Technology, Cambridge, Mass, USA, 1979.

[8] E. K. Boukas, " $H_{\infty}$ control of discrete-time Markov jump systems with bounded transition probabilities," Optimal Control Applications \& Methods, vol. 30, no. 5, pp. 477-494, 2009.

[9] M. Shen and G.-H. Yang, " $H_{2}$ state feedback controller design for continuous Markov jump linear systems with partly known information," International Journal of Systems Science, vol. 43, no. 4, pp. 786-796, 2012.

[10] X.-M. Sun, G.-P. Liu, D. Rees, and W. Wang, "Stability of systems with controller failure and time-varying delay," IEEE Transactions on Automatic Control, vol. 53, no. 10, pp. 2391-2396, 2008.

[11] L. Zhang and E.-K. Boukas, "Stability and stabilization of Markovian jump linear systems with partly unknown transition probabilities," Automatica, vol. 45, no. 2, pp. 463-468, 2009.

[12] L. Zhang and J. Lam, "Necessary and sufficient conditions for analysis and synthesis of Markov jump linear systems with incomplete transition descriptions," IEEE Transactions on Automatic Control, vol. 55, no. 7, pp. 1695-1701, 2010.

[13] Y. Zhang, Y. He, M. Wu, and J. Zhang, "Stabilization for Markovian jump systems with partial information on transition probability based on free-connection weighting matrices," Automatica, vol. 47, no. 1, pp. 79-84, 2011.
[14] C. Lin, Q.-G. Wang, and T. H. Lee, "A less conservative robust stability test for linear uncertain time-delay systems," IEEE Transactions on Automatic Control, vol. 51, no. 1, pp. 87-91, 2006.

[15] P. Park and J. W. Ko, "Stability and robust stability for systems with a time-varying delay," Automatica, vol. 43, no. 10, pp. 1855$1858,2007$.

[16] S. Xu, J. Lam, and X. Mao, "Delay-dependent $H_{\infty}$ control and filtering for uncertain Markovian jump systems with timevarying delays," IEEE Transactions on Circuits and Systems, vol. 54, no. 9, pp. 2070-2077, 2007.

[17] Y.-Y. Cao, J. Lam, and L. Hu, "Delay-dependent stochastic stability and $H_{\infty}$ analysis for time-delay systems with Markovian jumping parameters," Journal of the Franklin Institute, vol. 340, no. 6-7, pp. 423-434, 2003.

[18] G. Wang, Q. Zhang, and V. Sreeram, "Robust delay-rangedependent stabilization for Markovian jump systems with mode-dependent time delays and nonlinearities," Optimal Control Applications \& Methods, vol. 31, no. 3, pp. 249-264, 2010.

[19] Z. Wang, H. Qiao, and K. J. Burnham, “On stabilization of bilinear uncertain time-delay stochastic systems with Markovian jumping parameters," IEEE Transactions on Automatic Control, vol. 47, no. 4, pp. 640-646, 2002.

[20] H. K. Khalil, Nonlinear Systems, Prentice-Hall, Upper Saddle River, NJ, USA, 1996.

[21] X.-G. Guo and G.-H. Yang, "Delay-dependent reliable $H_{\infty}$ filtering for sector-bounded nonlinear continuous-time systems with time-varying state delays and sensor failures," International Journal of Systems Science, vol. 43, no. 1, pp. 117-131, 2012.

[22] Q.-L. Han, "Absolute stability of time-delay systems with sectorbounded nonlinearity," Automatica, vol. 41, no. 12, pp. 21712176, 2005. 


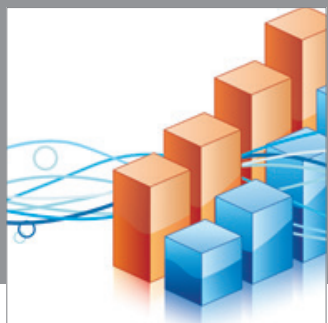

Advances in

Operations Research

mansans

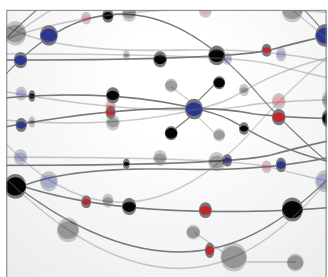

The Scientific World Journal
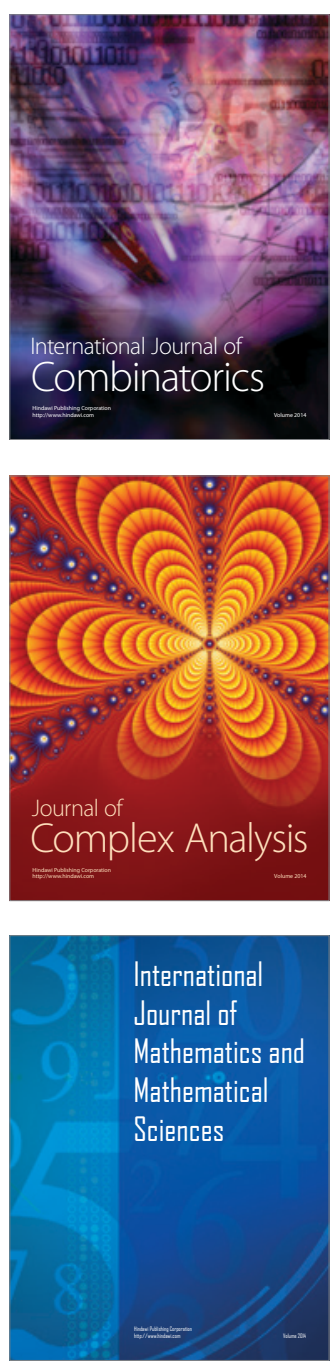
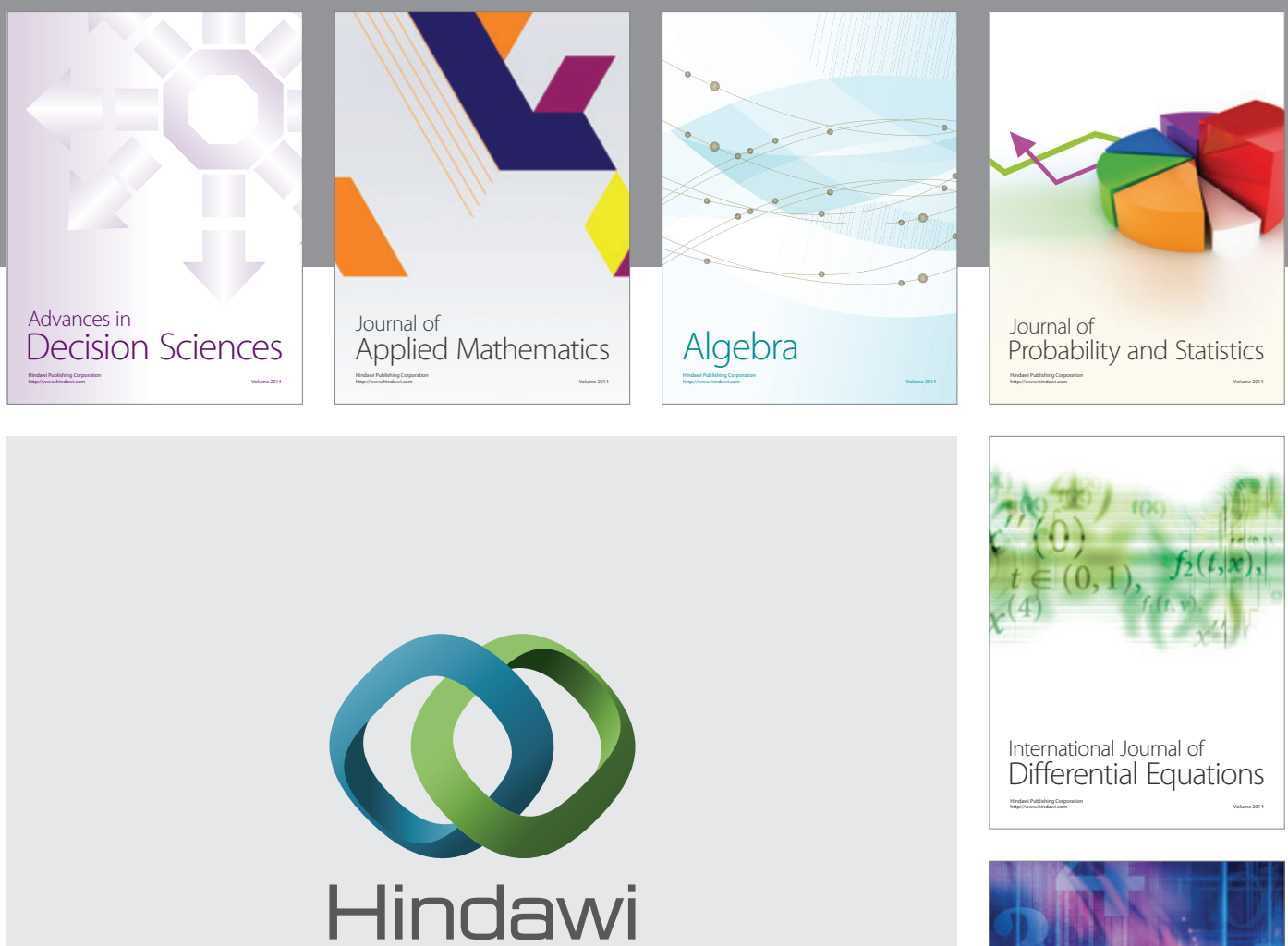

Submit your manuscripts at http://www.hindawi.com
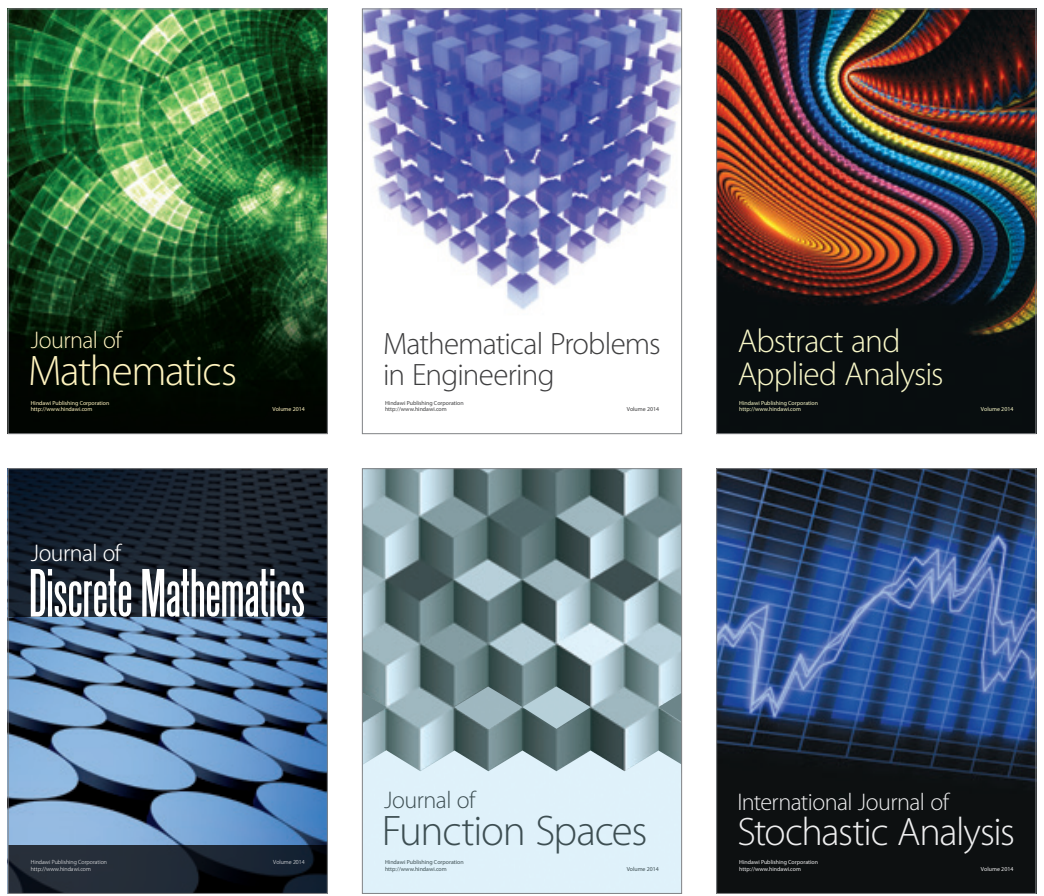

Journal of

Function Spaces

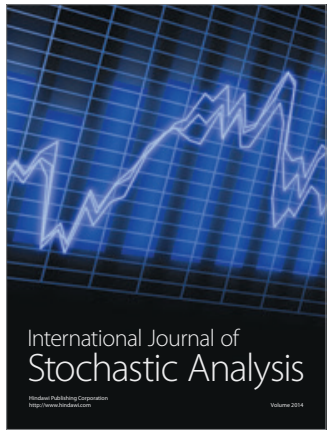

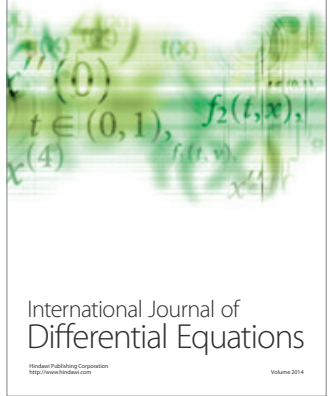
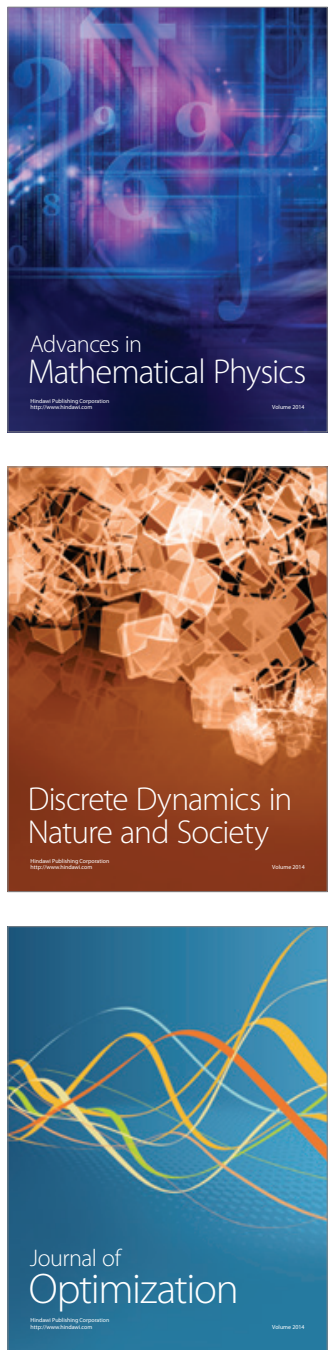